\title{
Perfil das malformações congênitas no berçário anexo à maternidade do Hospital das Clínicas da Universidade de São Paulo
}

\author{
Profile of the congenital malformations in a \\ Neonatal Intensive Care Unit (NICU) at a tertiary \\ care reference University Hospital in Brazil
}

\author{
Marina de Souza Pimenta1, Valdenise Martins Laurindo \\ Tuma Calil², Vera Lúcia Jornada Krebs ${ }^{3}$
}

\begin{abstract}
Pimenta MS, Calil VMLT, Krebs VLJ. Perfil das malformações congênitas no berçário anexo à maternidade do Hospital das Clínicas da Universidade de São Paulo. Rev Med (São Paulo). 2010 jan.-mar.;89(1):50-6.

RESUMO: OBJETIVO: Descrever a incidência e os tipos de malformações congênitas, a mortalidade e o tempo de permanência hospitalar de recém-nascidos (RN) portadores de malformações congênitas admitidos em berçário de alta complexidade de um hospital universitário. MÉTODOS: Estudo observacional retrospectivo e prospectivo de uma coorte de recém-nascidos vivos portadores de malformações congênitas. Foram incluídos todos os neonatos com malformação, nascidos no período de um ano (2007-2008) no Berçário Anexo à Maternidade do Hospital das Clínicas da Faculdade de Medicina da Universidade de São Paulo. Os dados foram obtidos por meio de análise de prontuários e avaliação dos RN. Para a descrição dos resultados, foram utilizadas a frequência relativa e absoluta das classes de cada variável qualitativa e medidas de tendência central e de dispersão (variáveis quantitativas). Na comparação entre as variáveis, foram usados o teste do qui-quadrado ou o teste exato de Fisher. RESULTADOS: Houve 1628 nascidos-vivos no período, sendo $93(5,71 \%)$ portadores de malformações. Predominaram as malformações neurológicas (33\%), urogenitais $(32 \%)$ e craniofaciais (31\%). Houve $46(49,5 \%)$ RN de termo e 47 (50,5\%) pré-termos; 41 (44,09\%) foram RN de baixo peso. Dentre os RN com malformações, 86 (92\%) apresentavam malformações maiores e 7 (8\%), malformações menores. O tempo médio de internação foi de 43,8 dias. Houve 21 (22,6\%) óbitos, com associação significante entre mortalidade e malformações ( $p<0,0001)$, sobretudo cardíacas $(p=0,014)$. CONCLUSÃO: A freqüência de malformações congênitas foi elevada em relação à descrita na literatura, havendo predomínio de malformações maiores. As anomalias mais comuns foram as neurológicas, conforme verificado por outros autores. Observou-se, também, uma mortalidade superior à encontrada na literatura, o que pode ser explicado pela gravidade dos casos descritos. A permanência hospitalar foi prolongada em relação à média dos recém-nascidos da unidade. Houve associação significante entre mortalidade e presença de malformações congênitas, principalmente cardíacas.
\end{abstract}

DESCRITORES: Anormalidades congênitas/epidemiologia; Recém-nascido; Mortalidade neonatal.

Estudo realizado no Berçário Anexo à Maternidade do Hospital das Clínicas da Faculdade de Medicina da Universidade de São Paulo. Pôster apresentado no "20ำ Medical and Nursing Congress Caring Without Borders", da European Society of Paediatric and Neonatal Intensive Care em Verona, Itália, de 14 a 17 de junho de 2009.

1. Acadêmica da Faculdade de Medicina da Universidade de São Paulo.

2. Doutora em Pediatria pela FMUSP, Médica Encarregada de Setor Técnico do Berçário Anexo à Maternidade do Hospital das Clínicas da Faculdade de Medicina da Universidade de São Paulo - HCFMUSP.

3. Livre Docente em Pediatria pela FMUSP, Chefe do Berçário Anexo à Maternidade do Hospital das Clínicas da Faculdade de Medicina da Universidade de São Paulo - HCFMUSP.

Endereço para correspondência: Marina de Souza Pimenta. Rua das Camélias, 357 - São Paulo, SP. ms.pimenta@ yahoo.com.br 


\section{INTRODUÇÃO}

$\mathrm{M}$ alformação congênita é qualquer alteração da estrutura anatômica normal presente ao nascimento ${ }^{12}$. A expressão "defeito congênito" tem, no entanto, significado mais amplo, incluindo toda anomalia funcional ou estrutural do desenvolvimento do feto decorrente de fatores pré-natais, sejam eles de origem genética, ambiental ou desconhecida; não é necessário que o defeito congênito esteja aparente ao nascimento, podendo se manifestar em época mais tardia ${ }^{16,18}$

O impacto dos defeitos congênitos no Brasil vem aumentando progressivamente ${ }^{7,8}$. Entre os anos de 1980 e 2000, as malformações ascenderam da quinta para a segunda posição como causa de óbitos em menores de um ano, apontando para a necessidade de estratégias específicas na política de saúde $8,10,18$. Estima-se que cerca de $5 \%$ dos nascidos vivos apresentem alguma anomalia do desenvolvimento ${ }^{7,16}$.

Uma pesquisa brasileira realizada pelo Instituo Fernandes Figueira, realizada a partir da análise de dados coletados entre janeiro de 1999 e julho de 2003, revelou que malformação congênita constituiu um dos três principais diagnósticos codificados na internação em $37 \%$ das admissões hospitalares pediátricas. A mortalidade hospitalar no grupo com malformação foi de $9,8 \%$, correspondendo ao dobro daquela observada no grupo sem malformação?

Além da elevada mortalidade, os recémnascidos com malformações congênitas apresentam maior morbidade, definida como risco para o desenvolvimento de complicações clínicas relacionadas ao número de internações e à gravidade das intercorrências ${ }^{7,8}$.

Apesar dos fatores genéticos estarem implicados em praticamente todas as doenças, como resultado de sua interação com o ambiente, seu papel relativo pode ser maior ou menor ${ }^{7,14}$. As doenças com componente genético preponderante incluem as monogênicas, que acometem $2 \%$ da população geral, as cromossômicas, presentes em $0,7 \%$ dos nascidos vivos e em metade dos abortamentos espontâneos, e ainda as multifatoriais, responsáveis por grande parcela das malformações congênitas ${ }^{8,11}$.

As malformações congênitas podem ser isoladas (únicas) ou múltiplas e podem ser classificadas como maiores ou menores ${ }^{1,17}$. Definese como malformação congênita maior ou "major" o defeito grave capaz de diminuir a expectativa de vida ou comprometer a função normal, necessitando de tratamento corretivo; trata-se de defeito que afeta a aceitação social do individuo ${ }^{11,17}$. Ocorre em $2 \%$ a $3 \%$ dos recém-nascidos vivos e tem ultrapassado a prematuridade como causa de mortalidade neonatal ${ }^{2,13}$.

O Berçário Anexo à Maternidade (BAM) do Hospital das Clínicas da Faculdade de Medicina da Universidade de São Paulo (HC-FMUSP) possui 63 leitos e recebe, em média, 200 recém-nascidos por mês. O Berçário admite, exclusivamente, recémnascidos de mães portadoras de patologias agudas ou crônicas, bem como de complicações obstétricas graves, sendo um centro de referência terciário para atendimento de recém-nascidos gravemente enfermos. Devido a essa característica, levantou-se a hipótese de que a frequência de recém-nascidos malformados no BAM seja elevada em relação à população geral, resultando na necessidade de uma infra-estrutura complexa para seu atendimento.

O estudo visa descrever a incidência e os tipos de malformações congênitas, a mortalidade e o tempo de permanência hospitalar de recémnascidos malformados admitidos em berçário de alta complexidade de um hospital universitário.

\section{CASUÍSTICA E MÉTODOS}

Trata-se de um estudo observacional retrospectivo e prospectivo de uma coorte de recém-nascidos vivos portadores de malformações congênitas. Foram incluídos todos os recémnascidos portadores de malformações congênitas diagnosticadas no período neonatal nascidos vivos no BAM no período de $1^{\circ}$ de agosto de 2007 a 31 de julho de 2008.

Os dados foram obtidos por análise de prontuários e avaliação dos recém-nascidos, com ênfase no exame físico detalhado e nos exames complementares indicados para elucidação diagnóstica.

O diagnóstico da malformação foi realizado no primeiro exame após o nascimento e confirmado durante a internação do recém-nascido. Os exames laboratoriais e de imagem foram efetuados conforme indicação clínica do neonatologista responsável e do geneticista.

O acompanhamento dos pacientes durante a internação incluiu reuniões conjuntas da equipe multiprofissional com os pais dos recém-nascidos.

Foi realizado um banco de dados incluindo as características físicas dos recém-nascidos afetados, os resultados dos exames complementares, as medidas terapêuticas necessárias para cada caso e a evolução clínica desses neonatos. As variáveis analisadas foram: peso ao nascer, idade gestacional, gênero, anormalidades observadas ao exame físico, 
Pimenta MS, et al. Perfil das malformações congênitas no Berçario anexo à maternidade.

resultados de cariótipos e de exames de imagem e tempo de permanência hospitalar.

Foram considerados recém-nascidos de termo aqueles com idade gestacional entre 37 e 41 semanas ${ }^{6,7}$. Definiu-se como mães adolescentes aquelas com idade igual ou inferior a 18 anos e como mães idosas aquelas com idade igual ou superior a 35 anos.

Considerou-se como malformação maior aquela com efeito adverso na saúde do indivíduo, em seu desempenho funcional ou em sua aceitação social. Nos casos de anomalias múltiplas, o mesmo paciente foi inserido simultaneamente em diferentes categorias de malformações.

Para a descrição dos resultados foram utilizadas as frequências relativas (percentuais) e absolutas ( $n$ ) das classes de cada variável qualitativa. A análise das variáveis quantitativas foi efetuada por medidas de tendência central (médias, medianas) e de dispersão (valor mínimo, valor máximo e desviopadrão). Foi utilizado o teste exato de Fisher para análise das variáveis. Valores de $\mathrm{p}<0,05$ foram considerados significantes.

O protocolo (no 00715200807) foi aprovado pela Comissão de Ética em Pesquisa da Instituição em 07/03/2008.

\section{RESULTADOS}

No período entre $1^{\circ}$ de agosto de 2007 e 31 de julho de 2008 houve 1628 nascidos vivos no BAM HC-FMUSP, dentre os quais 93 (6\%) eram portadores de malformações.

Dentre os 93 recém-nascidos com malformações, $46(49,5 \%)$ nasceram de termo e 47 $(50,5 \%)$, de pré-termo (Gráfico 1$)$.

Nove $(9,7 \%)$ dos recém-nascidos com malformações eram filhos de mães adolescentes e 26 (28\%) de mães idosas. Quarenta e sete (51\%) RN nasceram antes de 37 semanas de gestação (prétermo) e 46 (49\%) nasceram entre 37 e 42 semanas de gestação (termo).

Gráfico 1. Presença de malformação nos recém-nascidos no berçário anexo à maternidade do HCFMUSP

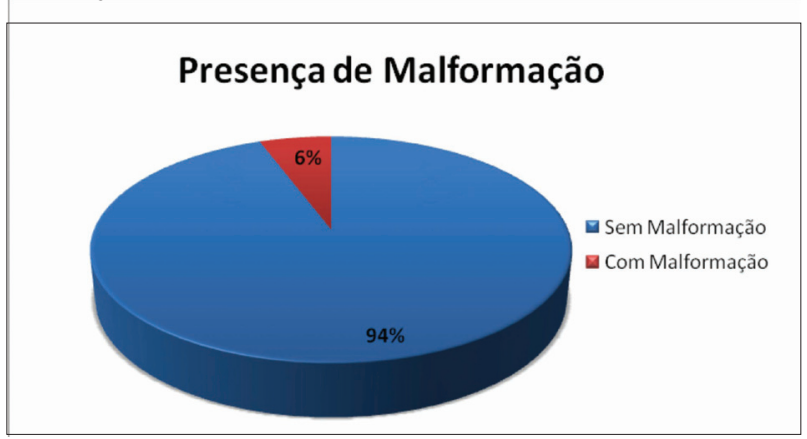

Setenta e nove (84\%) nasceram de parto operatório, nove (11\%) de parto normal e $5(5 \%)$ de parto com fórceps.

Quarenta e um neonatos foram de baixo peso (44\%), sendo 35 (38\%) com peso entre 1500 g e 2500 g e 6 (6\%) com peso entre $1000 \mathrm{~g} \mathrm{e} 1500 \mathrm{~g}$. Nenhum neonato apresentou peso ao nascer inferior a 1000g (Gráfico 2).

Cinquenta $(53,8 \%)$ recém-nascidos eram do gênero masculino, $41(44,1 \%)$ do gênero feminino e $2(2,1 \%)$ de gênero indeterminado.

Houve 10 gemelares $(10,53 \%)$, dentre os quais 2 pares de xifópagos $(4,22 \%$ do total de malformações).

Gráfico 2. Peso ao nascer dos recém-nascidos no berçário anexo à maternidade do HCFMUSP

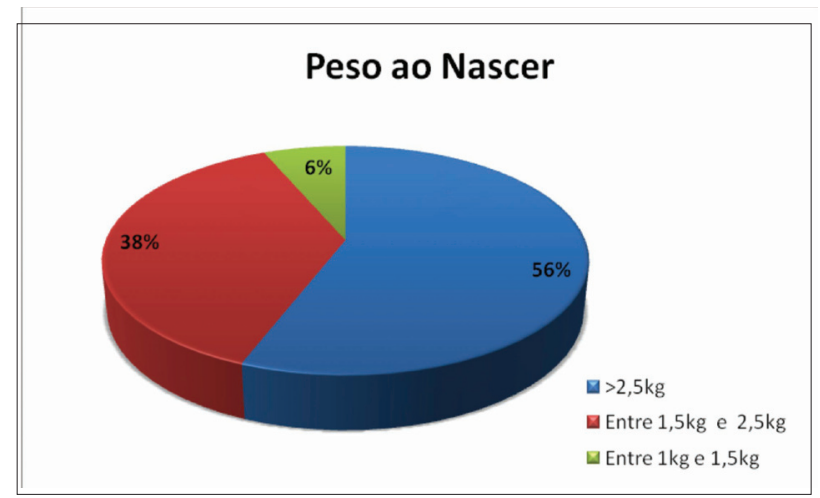

Cinquenta neonatos (52,63\%) apresentaram malformações isoladas e $44(46,32 \%)$ apresentaram malformações múltiplas (Gráfico 3). Oitenta e seis deles $(92 \%)$ apresentaram malformações maiores e $7(8 \%)$ apresentaram malformações menores (8\%) (Gráfico 4).

O tempo médio de internação foi de 43,8 dias. Vinte e dois neonatos $(23,66 \%)$ morreram, sendo que $18(19,35 \%)$ foram a óbito após 24 horas de vida e 4 $(4,30 \%)$ antes de 24 horas de vida (Gráfico 5).

Gráfico 3. Perfil das malformações nos recém-nascidosdo HCFMUSP

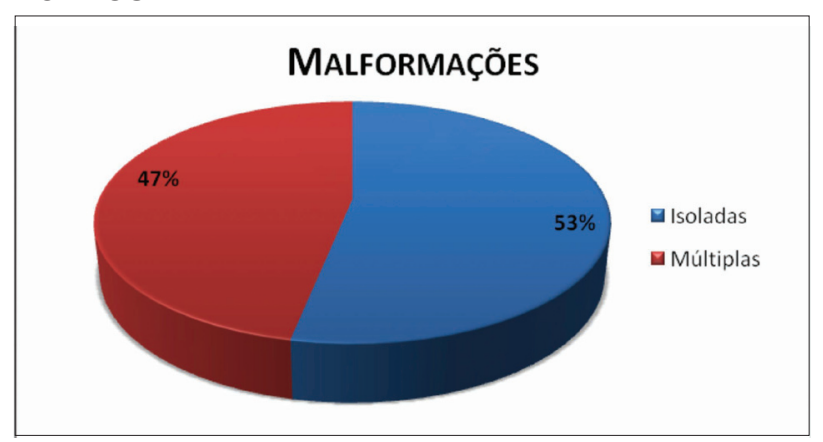


Gráfico 4. Perfil das malformações nos recém-nascidosdo HCFMUSP

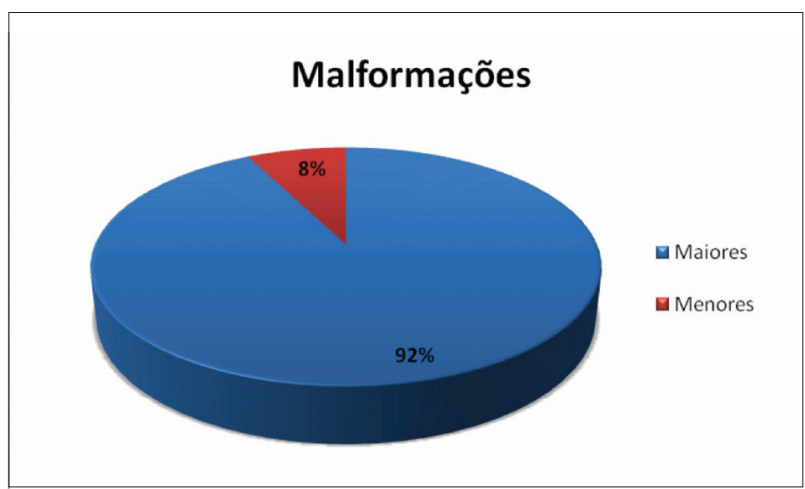

As malformações mais comuns foram as neurológicas, presentes em 31 (33,33\%) dos recém-nascidos analisados. As malformações geniturinárias ficaram em segundo lugar, afetando $30(32,26 \%)$ neonatos, seguidas pelas craniofaciais, presentes em $29(31,18 \%)$ deles. Dezenove $(20,43 \%)$ recém-nascidos apresentaram malformações osteoarticulares, $17(18,28 \%)$ apresentaram malformação da parede abdominal e $11(11,83 \%)$ apresentaram malformações cardíacas. Oito
Gráfico 5. Mortalidade dos recém-nascidos com malformações congênitas

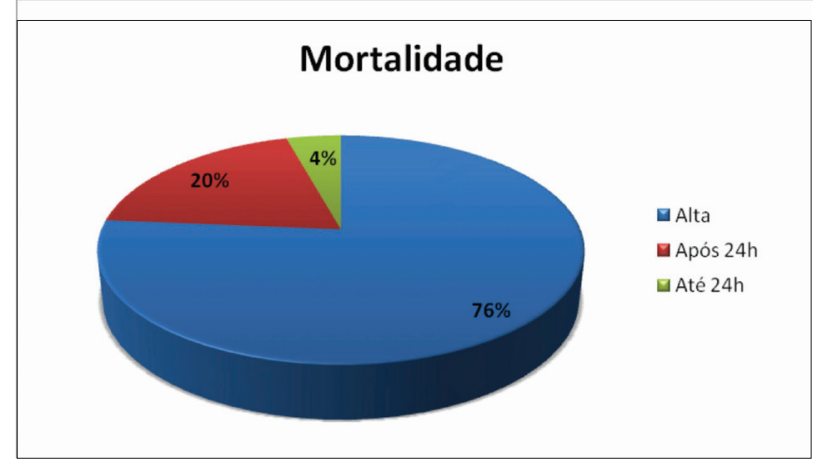

neonatos $(8,60 \%)$ eram portadores de síndromes genéticas, como a trissomia dos cromossomos 18 e 21 , sendo 7 deles $(7,53 \%)$ portadores de malformações gastrintestinais e $2(2,15 \%)$ de anomalias respiratórias (Figura 1).

A Tabela 1 mostra associação significante entre presença de malformação e mortalidade ( $p$ $<0,0001)$. Esta associação foi mais evidente na presença de malformação cardíaca, associada ou isolada $(p=0,014)$.

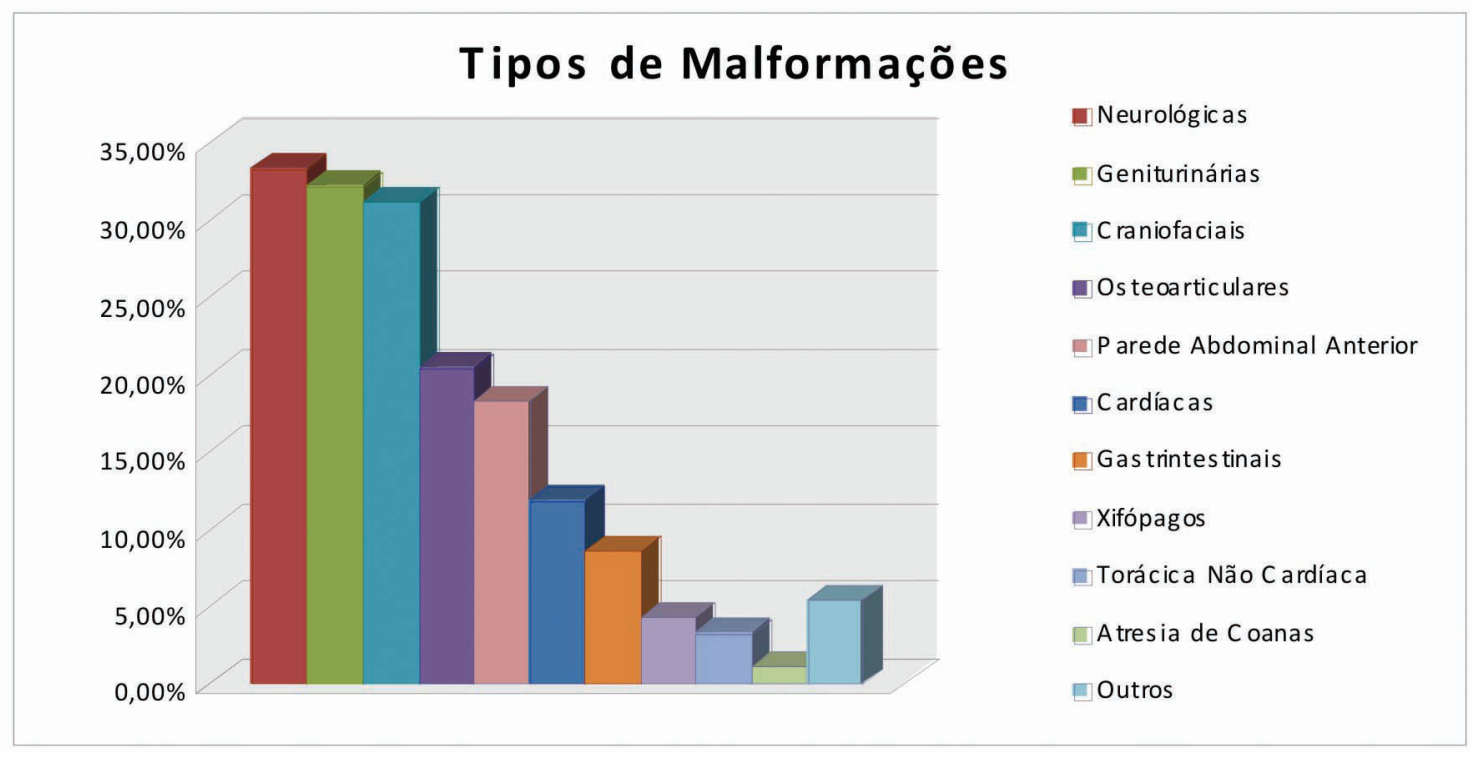

Figura 1. Tipos de malformações recém-nascidos no berçário anexo à maternidade do HCFMUSP 
Pimenta MS, et al. Perfil das malformações congênitas no Berçario anexo à maternidade.

Tabela 1. Associação entre presença de cada tipo de malformação e mortalidade*

\begin{tabular}{lcrrr}
\hline & Óbito & \multicolumn{1}{c}{ Alta } & \multicolumn{1}{c}{ Total } & p \\
\cline { 2 - 3 } Tipo de Malformação & $\mathbf{n}(\%)$ & $\mathbf{n}(\%)$ & $\mathbf{n}(\%)$ & \\
\hline Cardíaca & $6(6)$ & $5(5)$ & $11(12)$ & 0,014 \\
Neurológica & $5(5)$ & $26(28)$ & $31(33)$ & 0,430 \\
Geniturinária & $4(4)$ & $26(28)$ & $30(32)$ & 0,187 \\
Parede Abdominal Anterior & $6(6)$ & $11(12)$ & $17(18)$ & 0,200 \\
Gastrintestinal & $2(2)$ & $6(6)$ & $8(8)$ & 1,000 \\
Osteoarticular & $3(3)$ & $16(17)$ & $19(20)$ & 0,540 \\
Todos os tipos de Malformação & $22(24)$ & $71(76)$ & $93(100)$ & $<0,0001$ \\
\hline
\end{tabular}

*Recém-nascidos com malformações múltiplas foram inseridos em mais de uma categoria de malformação.

\section{DISCUSSÃO}

A importância das malformações congênitas como causa de óbito neonatal e pós-neonatal vem crescendo significativamente nos últimos anos. $\mathrm{Na}$ cidade de São Paulo, assim como no Brasil, constituem a segunda causa de morte em menores de um ano $8,10,18,20$. Nos países desenvolvidos, as anomalias congênitas são responsáveis por $20 \%$ da mortalidade neonatal e 30 a $50 \%$ da mortalidade perinatal $^{3}$.

No Instituto Fernandes Figueira ${ }^{17}$, durante o período de 2000 a 2004, a taxa de malformações congênitas entre os recém-nascidos foi de 2,84\%. Castro et al. ${ }^{3}$ relataram incidência de 2 a $3 \%$ nos países desenvolvidos. Estes autores encontraram, em estudo realizado em Pelotas (RS) entre os anos de 1990 e 2002, taxa de 1,37\% de anomalias congênitas. Amorim et al. ${ }^{2}$ descreveram incidência de 2,7\% de malformações congênitas entre os recémnascidos vivos em Recife, enquanto Horovitz et al. ${ }^{7}$ relataram taxa populacional de anomalias congênitas próxima de $5 \%$. No presente estudo, a incidência foi maior (6\%), pelo fato de se tratar de um berçário de alta complexidade. O BAM recebe casos do serviço de Medicina Fetal da Instituição, referência nacional para gestações de risco, incluindo aquelas de fetos portadores de malformações. Sabe-se que a presença de malformações congênitas associa-se freqüentemente à prematuridade e ao crescimento intrauterino restrito. Na casuística estudada, a taxa de prematuridade foi elevada (51\%), assim como o baixo peso ao nascer (44\%). Resultados semelhantes foram observados por Amorim et al, que encontraram taxas de prematuridade e de baixo peso ao nascer de $55 \%$ e $50,4 \%$, respectivamente, entre recém-nascidos malformados ${ }^{2}$.

No presente estudo houve prevalência de malformações maiores, principalmente neurológicas $(33,3 \%)$, geniturinárias $(32,3 \%)$ e craniofaciais $(31,2 \%)$, concordando com outros autores ${ }^{2}$, que descreveram maior prevalência de malformações neurológicas $(27,4 \%)$.

Foi encontrada associação altamente significante entre mortalidade e presença de malformação congênita ( $p<0,0001)$. Quando as malformações foram separadas por tipo, houve associação significante apenas entre malformações cardíacas e mortalidade, sugerindo um pior prognóstico dessas anomalias. A associação entre mortalidade e malformações congênitas já era esperada, assim como o prolongado tempo de internação desses recém-nascidos, devido ao seu maior risco. A mortalidade encontrada neste estudo foi de $24 \%$, taxa extremamente alta se comparada à encontrada em 2003 no Instituto Fernandes Figueira $(9,8 \%)^{9}$. Esse dado pode ser explicado pela maior complexidade dos casos atendidos no serviço, onde $47 \%$ das malformações eram múltiplas, enquanto Amorim et al. ${ }^{2}$ e Castro et al. ${ }^{3}$ relataram apenas $13,3 \%$ e $8,1 \%$ de anomalias múltiplas, respectivamente. A mortalidade encontrada no presente estudo nas primeiras 24 horas de vida foi de 4,3\%, taxa elevada, considerando que foram excluídos do cálculo os natimortos e os que faleceram ainda na sala de parto. Esse dado ilustra a gravidade dos recém-nascidos malformados admitidos no BAM.

A análise final dos dados obtidos mostra que a frequência de malformações congênitas no BAM foi elevada, em relação à descrita na literatura, havendo predomínio de malformações maiores. A permanência hospitalar foi prolongada em relação à média dos recém-nascidos da unidade. As anomalias mais comuns foram as do sistema nervoso 
central, do trato geniturinário e craniofaciais. Houve associação significante entre mortalidade e presença de malformações congênitas, principalmente cardíacas.

AGRADECIMENTO: Este trabalho foi realizado com o auxílio da Fundação de Amparo à Pesquisa do Estado de São Paulo (FAPESP).

Pimenta MS, Calil VMLT, Krebs VLJ. Profile of the congenital malformations in a Neonatal Intensive Care Unit (NICU) at a tertiary care reference University Hospital in Brazil. Rev Med (São Paulo). 2010 jan.-mar.;89(1):50-6.

\begin{abstract}
OBJECTIVE: To describe the incidence and type of congenital malformation (CM), hospital mortality and amount of time in hospital of newborn babies (NB) with CM admitted to a neonatal intensive care unit (NICU) in a tertiary care reference university hospital in Brazil. METHODS: Observational retrospective and prospective study of a cohort of NB with CM. We studied all NB with CM in the period of one year (2007-2008) borned in the Bercário Anexo à Maternidade do Hospital das Clínicas da Faculdade de Medicina da Universidade de São Paulo. The data was collected from clinical records and NB avaliation. Results were described by relative and absolute frequency of the classes of each qualitative variable and measures of central tendency and dispersion (quantitative variables). Chi-square and the Fisher's exact tests were used to compare the variables. RESULTS: There were 1628 NB in the period, $93(5.71 \%)$ of which had CM. The most prevalent CM were neurological $31(33 \%)$, genitourinary $30(32 \%)$ and craniofacial 29 (31\%). Among NB with CM, 46 (49.5\%) were term and $47(50.5 \%)$ were preterm. Low birth weight was observed in $41(44.09 \%)$ of NB. Major and minor malformations were diagnosed in $86(92 \%)$ and $7(8 \%)$ of NB, respectively. The average time of hospitalization was 43.8 days and $21(22.6 \%)$ of NB with CM died. There was a significant association between mortality and CM $(p<0.0001)$, specially cardiac malformations $(p=0.014)$ CONCLUSION: The prevalence of $\mathrm{CM}$ was higher than found in literature, major malformation being more prevalent. The majority of CM was neurological, as found by others authors. Compared to literature, the NB with CM studied had higher mortality and longer period of hospitalization, what can be explained by the gravity of the cases. There was significant association between $\mathrm{CM}$ and mortality, mainly due to cardiac malformations.
\end{abstract}

KEY WORDS: Congenital abnormalities/epidemiology; Infant, newborn; Neonatal mortality (Public health).

\title{
REFERÊNCIAS
}

1. Aguiar MJB, Pena SDJ. O recém-nascido com malformações e sua família. In: Alves Filho N, Corrêa MD, Alves Jr JMS, Corrêa Jr MD, editores. Perinatologia básica. 3a ed. Rio de Janeiro: Medsi/Guanabara Koogan; 2006. p.122-8.

2. Amorim MMR, Vilela PC, Santos ARVD, Lima ALMV, Melo EFP, Bernardes HF, et al. Impacto das malformações congênitas na mortalidade perinatal e neonatal em uma maternidade-escola do Recife. Rev Bras Saúde Matern Infant (Recife). 2006;6(Supl 1):S19-S25.

3. Castro MLS, Cunha CJ, Moreira PB, Fernández RR, Garcias GL, Martino-Röth MG. Freqüência das malformações múltiplas em recém-nascidos na Cidade de Pelotas, Rio Grande do Sul, Brasil, e fatores sócio-demográficos associados. Cad Saúde Pública. 2006;22(5):1009-15.
4. DATASUS, 2007. Disponível em: http://tabnet.datasus. gov.br/cgi/tabcgi.exe? sim/cnv/infsp.def

5. Departamento de Informação e Documentação, Instituto Fernandes Figueira, Fundação Oswaldo Cruz. Rio de Janeiro; 2003.

6. Dulskiene V, Malinauskiene V, Azaraviciene A, Kuciene $R$. The incidence and diagnostics of congenital heart defects in Kaunas infant population during 1999-2005. Medicina (Kaunas). 2008;44(2):139-46.

7. Horovitz DDG, Cardoso MHCA, Llerena Jr JC, Mattos RA. Atenção aos defeitos congênitos no Brasil: características do atendimento e propostas para formulação de políticas públicas em genética clínica. Cad Saúde Pública. 2006;22(12):2599-609. 
Pimenta MS, et al. Perfil das malformações congênitas no Berçario anexo à maternidade.

8. Horovitz DDG, Llerena Jr. JC, Mattos RA. Atenção aos defeitos congênitos no Brasil: panorama atual. Cad Saúde Pública. 2005;21(4):1055-64.

9. Instituto Fernandes Figueira, Fundação Oswaldo Cruz. Boletim de produção de serviços. Arquivo nosológico gerado em 28/Ago/2003. Rio de Janeiro:

10. Kirby RS, Seaver LH. Birth defects research: improving surveillance methods and addressing epidemiologic questions and public health issues. Birth Defects Res A Clin Mol Teratol. 2005;73(10):645.

11. Lansky S, França E, Leal MC. Mortalidade perinatal e evitabilidade: revisão da literatura. Rev Saúde Pública. 2002;36(6):759-72.

12. McLean SD. Congenital anomalies. In: MacDonald MG, Seshia MMK, Mullett MD, edityors. Avery's neonatology. Pathophysiology \& management of the newborn. 6th ed. Philadelphia: Lippincott Williams and Wilkins; 2005. p.892-913.

13. Nelson K, Holmes LB. Malformations due to presumed spontaneous mutations in newborn infants. $\mathrm{N}$ Engl J Med. 1989;320(1):19-23.

14. Nussbaum RL, Mclnnes RR, Willard HF. Introduction - classification of genetic disorders. In: Thompson \&
Thompson genetics in medicine. 6th ed. Philadelphia: Saunders; 2001. p.2-3.

15. O'Connor M, McDaniel N, Brady WJ. The pediatric electrocardiogram part III: Congenital heart disease and other cardiac syndromes. Am J Emerg Med. 2008;26(24):497-503.

16. Organização Pan-Americana da Saúde. Prevenção e controle de enfermidades genéticas e os defeitos congênitos: relatório de um grupo de consulta. Washington (DC): OPS; 1984. (Publicação científica, 460).

17. Pacheco SS, Souza AI, Vidal AS, Guerra GVQL, Filho $M B$, Baptista EVP, et al. Prevalência dos defeitos de fechamento do tubo neural em recém-nascidos do Centro de Atenção à Mulher do Instituto Materno Infantil Prof. Fernando Figueira, IMIP: 2000-2004. Rev Bras Saúde Matern Infant (Recife). 2006;6(Supl 1):535-42.

18. Ramos JLA, Calil VMLT, Vaz FAC, Araújo J, Zuccolotto $M$, Corradini HB, et al. Malformações congênitas: estudo prospectivo de dois anos em três maternidades de São Paulo. Pediatria (S. Paulo). 1981;3:20-8.

19. Stoll C, Alembik Y, Dott B, Roth MP. Omphalocele and gastroschisis and associated malformations. Am J Med Genet A. 2008;146(10):1280-85. 